Article

\title{
Genomic Analysis of Clostridium perfringens BEC/CPILE-Positive, Toxinotype D and E Strains Isolated from Healthy Children
}

\author{
Raymond Kiu ${ }^{1}$, Kathleen Sim ${ }^{2}$, Alex Shaw ${ }^{2}$, Emma Cornwell ${ }^{2}$, Derek Pickard ${ }^{3}$, \\ J. Simon Kroll ${ }^{2}$ and Lindsay J. Hall ${ }^{1, *(D)}$ \\ 1 Gut Microbes \& Health, Quadram Institute Bioscience, Norwich NR4 7UQ, UK; \\ Raymond.Kiu@quadram.ac.uk \\ 2 Faculty of Medicine, Imperial College London, London W2 1NY, UK; K.Sim@imperial.ac.uk (K.S.); \\ a.shaw@imperial.ac.uk (A.S.); emma.zabaleta@hotmail.com (E.C.); s.kroll@imperial.ac.uk (J.S.K.) \\ 3 Department of Medicine, University of Cambridge, Cambridge CB2 0QQ, UK; djjp2@medschl.cam.ac.uk \\ * Correspondence: Lindsay.Hall@quadram.ac.uk
}

Received: 29 August 2019; Accepted: 16 September 2019; Published: 19 September 2019

check for updates

\begin{abstract}
Clostridium perfringens toxinotype D, toxinotype E, and gastroenteritis-linked BEC/CPILE-positive strains have never been reported in healthy children. We isolated, whole-genome sequenced and bioinformatically characterised three $C$. perfringens isolates-type D (IQ1), type E (IQ2) and BEC/CPILE-positive (IQ3), recovered from the stools of three healthy two-year-olds, which were further compared to $128 \mathrm{C}$. perfringens genomes available from NCBI. The analysis uncovered a previously under-described putative toxin gene alv (alveolysin) encoded by isolates IQ2 and IQ3, which appeared to be a clade-specific trait associated with strains from domestic animals. A plasmid analysis indicated that the iota-toxin was encoded on a near-intact previously described plasmid pCPPB-1 in type E strain IQ2. The BEC genes $b e c A$ and $b e c B$ were carried on a near-identical pCPOS-1 plasmid previously associated with Japanese gastroenteritis outbreaks. Furthermore, a close phylogenetic relatedness was inferred between the French C. perfringens type E isolates cp515.17 and newly sequenced IQ2, suggesting geographical links. This study describes novel C. perfringens isolates from healthy individuals which encode important toxin genes, indicating the potential spread of these veterinary and clinically important strains and mobile genetic elements, and highlights areas for future research.
\end{abstract}

Keywords: Clostridium perfringens; epsilon toxin; iota toxin; whole genome sequencing; infants; binary toxins; genome analysis

Key Contribution: These novel Clostridium perfringens toxigenic strains, including BEC-positive (or, CPILE), type D (carrying epsilon toxin) and type E (carrying iota toxin) isolates, are the first reported in healthy children. The strains were characterised by whole genome sequencing, which revealed further toxin genes, including $p f o A$ (perfringolysin $\mathrm{O}$ ), alv (alveolysin) and cpe (enterotoxin CPE). By applying multiple in silico approaches, we also reconstructed and investigated C. perfringens toxin-encoded plasmids, and a further phylogenetic analysis on $132 \mathrm{C}$. perfringens genomes enabled us to probe the linkage between $C$. perfringens toxinotypes and phylogenomic relatedness.

\section{Introduction}

Clostridium perfringens is a fast-growing Gram positive, anaerobic and spore-forming pathogen known to produce $>20$ toxins. These toxins induce various disease states in both humans and animals including gastroenteritis, gas gangrene, necrotic enteritis, necrotising enterocolitis, and 
enterotoxaemia [1-4]. Typically, based on the combinations of typing toxins that are produced, including alpha toxin, beta toxin, epsilon toxin and iota toxin, C. perfringens strains were classified into 5 distinct toxinotypes. More recently, this typing scheme has been updated to 7 toxinotypes (including NetB and enterotoxin CPE) to better reflect clinical relevance (toxinotypes A-G) [5,6].

C. perfringens toxinotype D isolates, capable of producing alpha toxin (encoded by plc) and epsilon toxin (etx), are known to cause natural enterotoxaemia (also commonly known as 'pulpy kidney') primarily in sheep, goats, and occasionally cattle [7-9]. Enterotoxaemia in sheep is characterised by sudden death syndrome, brain lesions, lung oedema and enterocolitis. The absorption of the epsilon toxin followed by bloodstream circulation to internal organs such as the brain and kidney results in an elevated blood pressure and neurological disease [10-13]. Epsilon toxin is also known as the third-most potent toxin after the botulinum and tetanus toxins [14], with the epsilon toxin gene etx typically encoded on plasmids [15].

C. perfringens type E isolates can produce alpha toxin and iota toxin (encoded by the iap and ibp genes) and may also carry secondary toxin genes such as cpb2 (beta2 toxin). These strains are primarily associated with diseases in domestic animals including enterotoxaemias in rabbits, and enteritis in lambs and cattle $[16,17]$. The binary iota toxin genes iap (encodes the enzymatic component) and $i b p$ (the binding component) are both plasmid-encoded [18].

More recently, it was reported that a potent binary enterotoxin of C. perfringens (BEC; also, CPILE), encoded by $\operatorname{bec} A$ and $\operatorname{bec} B$ genes, was linked to food poisoning outbreaks in Japan, with its enterotoxic activity confirmed experimentally $[19,20]$. This enterotoxin, distinct from typical gastroenteritis-associated enterotoxin CPE, as found to be carried on a $\sim 54 \mathrm{~kb}$ plasmid, suggesting its potential for horizontal gene transfer [19].

In this study, we performed comparative genomics to analyse C. perfringens strains obtained from healthy two-year-old children who had been born prematurely. Two of the isolates were characterised as the 'typical' veterinary-associated toxinotypes D (IQ1) and E (IQ2), respectively, and one was a BEC-positive strain (IQ3). This study contributes further understanding of toxin genes encoded in C. perfringens isolates from otherwise healthy individuals, including epsilon toxin, iota toxin and perfringolysin O. Furthermore, the type E and BEC-positive strains were also found to carry a rarely-discussed toxin-alveolysin—which despite sharing a high homology ( $~ 86 \%$ nucleotide identity) with perfringolysin $\mathrm{O}$, had distinct genetic sequences. A further in-depth analysis on toxin-encoding plasmids in toxinotype $\mathrm{D}$, toxinotype $\mathrm{E}$ and BEC-positive $C$. perfringens strains provided additional insights into the phylogenetic relatedness of these strains.

\section{Results}

\subsection{Genomic Description of Newly Sequenced C. perfringens Isolates}

In this study, we isolated and sequenced three C. perfringens strains from preterm-born healthy children who were originally recruited as part of the two-year NeoM (Neonatal Microbiota) study (Table S1 and Table 1). These three isolates were sequenced at an ultra-high coverage $(195 \times-212 \times)$, which enabled us to obtain high quality low-contig short-read sequenced draft genome assemblies at a high N50; IQ2 (15 contigs, N50: 2.1 mb), IQ3 (20 contigs, N50: 2.2 mb) and IQ1 (41 contigs, N50: $\sim 450 \mathrm{~kb}$ ). These genomes were taxonomically confirmed to be C. perfringens at a species level using various genomic approaches, including the $16 \mathrm{~S}$ rRNA gene, Average Nucleotide Identity (ANI) and digital DNA-DNA Hybridisation (dDDH). The GC percentages (\%) are stable between the genomes (range: $27.98 \%-28.03 \%$ ) and were predicted to contain an average of $\sim 3272$ genes per genome. These three C. perfringens strains were toxinotyped as type D (IQ1; encoding epsilon toxin), type E (IQ2; encoding iota toxin) and type A (IQ3; carrying BEC) respectively. A bioinformatics analysis of antimicrobial genes indicated the presence of only tet $A(P)$ in isolates IQ2 and IQ3. 
Table 1. Genome information and statistics of three newly sequenced C. perfringens isolates described in this study.

\begin{tabular}{cccc}
\hline Strain/Genome & $\begin{array}{c}\text { C. perfringens } \\
\text { IQ1 }\end{array}$ & $\begin{array}{c}\text { C. perfringens } \\
\text { IQ2 }\end{array}$ & $\begin{array}{c}\text { C. perfringens } \\
\text { IQ3 }\end{array}$ \\
\hline Genome size (bp) & $3,563,782$ & $3,438,840$ & $3,518,141$ \\
Sequencing coverage & $195 \times$ & $216 \times$ & $212 \times$ \\
Sequencing platform & Illumina HiSeq 2500 & Illumina HiSeq 2500 & Illumina HiSeq 2500 \\
No. of contigs & 41 & 15 & 20 \\
Genes & 3320 & 3199 & 3299 \\
CDS & 3219 & 3097 & 3196 \\
tRNAs & 91 & 92 & 92 \\
N50 length (bp) & 452,560 & $2,192,929$ & $2,217,274$ \\
GC $(\%)$ & 27.98 & 28.03 & 28.00 \\
ANI (\%) 1 & 98.35 & 96.89 & 97.01 \\
dDDH $(\%)$ & 87.40 & 74.50 & 75.30 \\
Toxinotype & D & E & A (BEC-positive) \\
ENA sample accession & SAMEA5818795 & SAMEA5818796 & SAMEA5818797 \\
ENA assembly accession & GCA_902459455 & GCA_902459435 & GCA_902459425 \\
\hline 1 The Average Nucleotide Identity (ANI) and digital DNA-DNA Hybridisation (dDDH) were calculated with \\
respect to the C. perfringens type strain ATCC13124 genome for species validation at a minimum cutoff of 95\% and \\
70\%, respectively.
\end{tabular}

\subsection{Comparisons of Toxin Gene Sequences}

Several important toxin gene sequences were compared, including; etx (epsilon-toxin), iap (iota-toxin component a), ibp (iota-toxin component b), becA (binary enterotoxin component a), becB (binary enterotoxin component $\mathrm{b}$ ), $p f o A$ (thiol-activated toxin perfringolysin $\mathrm{O}$ ) and alv (thiol-activated toxin alveolysin), as demonstrated in Tables $2-4$.

Table 2. Nucleotide sequence similarity comparison between multiple epsilon-toxin gene etx variants extracted from the toxinotype B and D C. perfringens genomes available in the NCBI databases.

\begin{tabular}{cccccccc}
\hline \multirow{2}{*}{ Strain } & \multirow{2}{*}{ Type } & \multicolumn{2}{c}{ SNP } & \multicolumn{5}{c}{ Sequence Similarity (\%) } \\
\cline { 3 - 7 } & & Pos.726 & ATCC3626 & JGS1721 & NCTC8503 & IQ1 & Type D \\
\hline ATCC3626 & B & A & 100.00 & 99.90 & 99.90 & 99.90 & 100.00 \\
JGS1721 & D & G & & 100.00 & 100.00 & 100.00 & 99.90 \\
NCTC8503 & D & G & & & 100.00 & 100.00 & 99.90 \\
IQ1 & D & G & & & & 100.00 & 99.90 \\
Type D & D & A & & & & & 100.00 \\
\hline
\end{tabular}

Table 3. Nucleotide sequence similarity pair-wise comparison between multiple iota-toxin genes iap and $i b p$ variants extracted from the various toxinotype E C. perfringens genomes.

\begin{tabular}{ccccccccc}
\hline \multirow{2}{*}{ Strain } & \multicolumn{3}{c}{ iap Sequence Similarity (\%) } & \multicolumn{4}{c}{ ibp Sequence Similarity (\%) } \\
\cline { 2 - 9 } & cp508.17 & cp515.17 & JGS1987 & IQ2 & cp508.17 & cp515.17 & JGS1987 & IQ2 \\
\hline cp508.17 & 100.00 & 99.85 & 90.40 & 99.85 & 100.00 & 99.96 & 88.44 & 99.96 \\
cp515.17 & - & 100.00 & 90.40 & 100.00 & - & 100.00 & 88.48 & 100.00 \\
JG51987 & - & - & 100.00 & 90.40 & - & - & 100.00 & 88.48 \\
IQ2 & - & - & - & 100.00 & - & - & - & 100.00 \\
\hline
\end{tabular}


Table 4. Nucleotide sequence similarity pair-wise comparison between multiple thiol-activated toxins including perfringolysin O gene $p f o A$ variants and the alveolysin gene alv (from strain IQ2 and IQ3) computationally extracted from various $C$. perfringens genomes.

\begin{tabular}{cccccccc}
\hline & & \multicolumn{5}{c}{ Average Nucleotide Sequence Similarity (\%) } \\
\cline { 3 - 8 } Strain & Gene & ATCC13124 & IQ1 & IQ2 & IQ3 & IQ2 & IQ3 \\
\cline { 3 - 7 } & & $p f o A$ & $p f o A$ & $p f o A$ & $p f o A$ & alv & alv \\
\hline \multirow{2}{*}{ ATCC13124 } & $p f o A$ & 100.00 & 98.87 & 97.54 & 97.34 & 86.62 & 86.82 \\
IQ1 & $p f o A$ & - & 100.00 & 97.34 & 97.14 & 86.62 & 86.62 \\
IQ2 & $p f o A$ & - & - & 100.00 & 99.67 & 86.62 & 86.62 \\
IQ3 & $p f o A$ & - & - & - & 100.00 & 86.36 & 86.56 \\
IQ2 & $a l v$ & - & - & - & - & 100.00 & 99.43 \\
IQ3 & alv & - & - & - & - & - & 100.00 \\
\hline
\end{tabular}

Epsilon-toxin gene etx variants appeared to be well conserved among 5 different $C$. perfringens strains at the nucleotide level, with only one different nucleotide identified at position 726 (987 bp alignment), which did not alter the amino acid residue serine (S) at position 254 (Table 2). The binary iota-toxin gene components iap and ibp were investigated in 4 toxinotype $\mathrm{E}$ strains, including the recently published French strains cp508.17 and cp515.17 [21]. Iota-toxin gene variants were shown to be highly conserved at a nucleotide identity $>99.85 \%$ (only one variable amino acid at position 134 , either isoleucine or threonine) among strains cp508.17, cp515.17 and IQ2 (Table 3). Notably, strain JGS1987 had more dissimilar iap and ibp components, assuming functional changes at the amino acid level ( 142 amino acid residue differences compared to IQ2; Table S2). The BEC-toxin gene components $\operatorname{bec} A$ and $b e c B$ were identical (100\% identity) to the reference bec $A B$ genes (Table S3).

A thiol-activated membrane-damaging toxin alveolysin gene alv, first described in Paenibacillus alvei, was detected in the C. perfringens isolates IQ2 (type E) and IQ3 (BEC-positive; Table S4) [22]. This gene is $1578 \mathrm{bp}$ long, with the alveolysin protein comprising 528 amino acids. At a nucleotide level, it shares 99.62\% similarity (IQ3 alv) with a similar gene in JP838 (accession CP010994.1; NCBI NR database) at 100\% query coverage, annotated as alveolysin (nucleotide identity: 100\%). In addition, this gene is similar to, but distinct ( $\sim 86 \%$ identity) from, typical perfringolysin $O$ gene $p f o A$ variants in C. perfringens at the nucleotide level (PfoA is a frequently-detected thiol-activated toxin produced by $C$. perfringens) (Table 4). A protein sequence search (blastp) on the NCBI non-redundant protein sequences database showed that alv shared a sequence homology with alv genes in several Gram-positive bacteria, including Paenibacillus alvei ( 74\%), Paeniclostridium sordellii ( 78\%), Paraclostridium bifermentans $(\sim 77 \%)$, Clostridium argentinense ( $78 \%)$, and Bacillus luciferensis ( 70\%).

\subsection{Comparative Study of Toxin-Encoding Plasmids}

Key C. perfringens-derived toxin genes are known to be carried on plasmids, including epsilon toxin, iota toxin and BEC $[19,23,24]$. Thus, we attempted to computationally reconstruct and investigate toxin-encoding plasmids using both a reference-based assembly (based on NCBI plasmid sequences) and de novo assembly-graph approaches (by extracting connected circular contigs from assembly graphs that represent potential plasmid sequences). Plasmid replication genes (ori) were also confirmed in these extracted sequences to validate our investigations (Figure 1). 

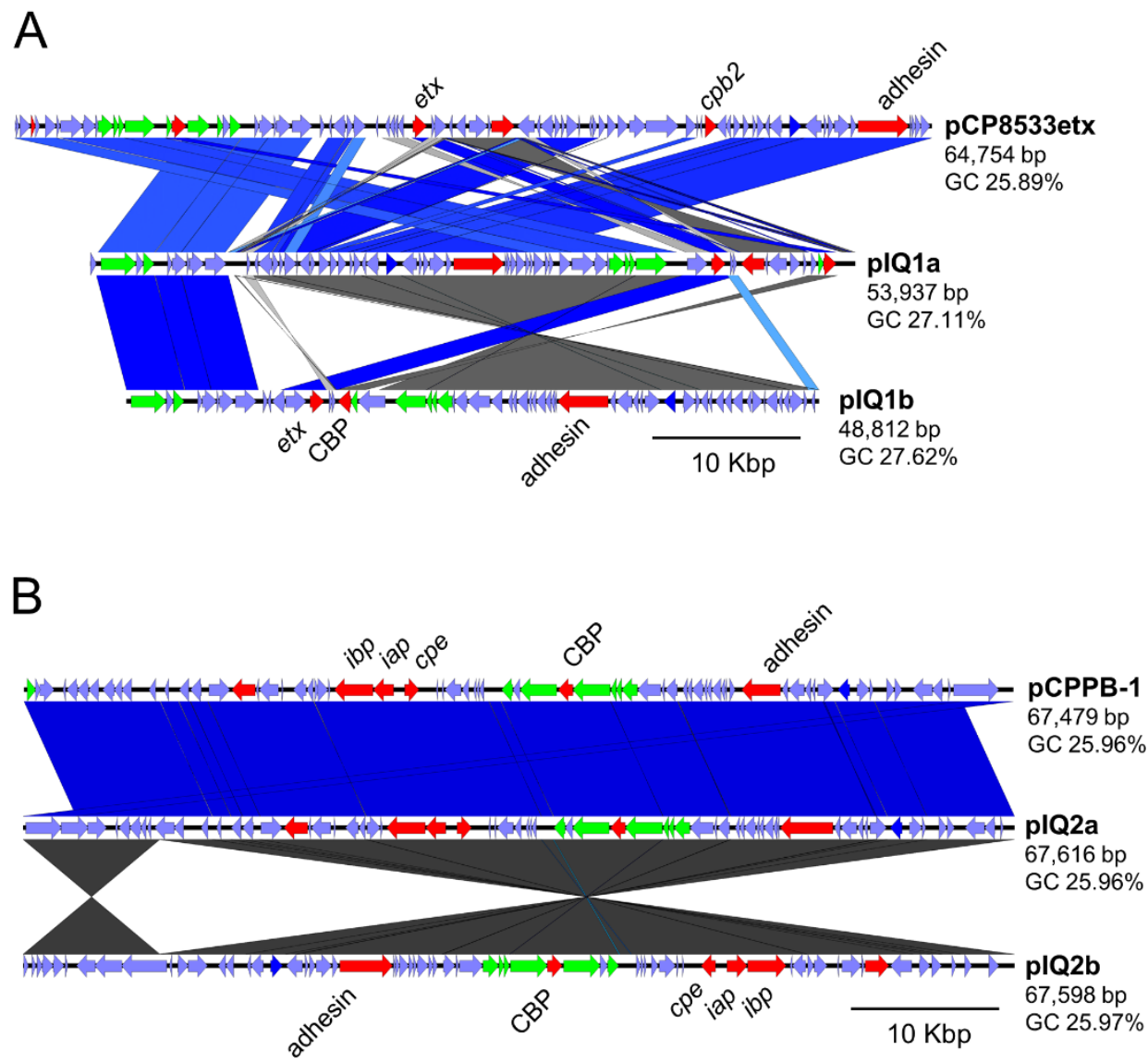

C

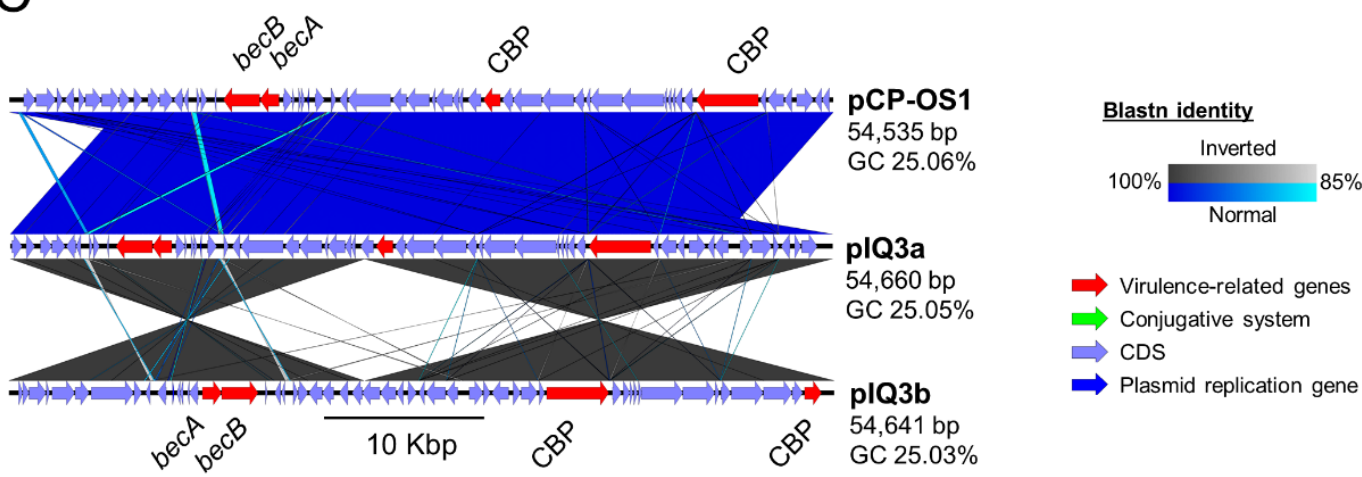

Figure 1. Plasmid sequence identity comparison maps showing the similarity of the reference plasmids with the computationally predicted toxin-encoding plasmids present in C. perfringens strains (A) IQ1, (B) IQ2, and (C) IQ3. In each sequence map, the reference plasmid sequence (top) was compared with the plasmid sequences reconstructed using the reference-based approach (middle) and assembly graph approach (bottom). CBP: Cell-wall Binding Protein.

Initially, pIQ1a was assembled in 13 contigs; length of 53,937 bases out of 64,753 bases based upon the only etx-reference plasmid pCP8533etx (from a lamb dysentery type B isolate in 1953; NCTC8533) available in the NCBI nucleotide database [25]. This suggested that pIQ1a was not the same as pCP8533etx (Figure 1A; Table 5), which was further supported by an unbiased assembly-graph approach (based on comparing the contig connection and sequencing depths: $\sim 188 \mathrm{X}$ in plasmids vs. $<50 \mathrm{X}$ in chromosomes). This analysis predicted that the circular plasmid pIQ1b was smaller $(\sim 48 \mathrm{~kb})$ 
than pCP8533etx and encoded $e t x$, but not $c p b 2$ as in pCP8533etx. This finding agrees with a previous study that reported that the etx-plasmid in cpe-negative $c p b 2$-negative type D C. perfringens strains was typically $\sim 48 \mathrm{~kb}$ long, indicating that the et $x$-plasmid pIQ1b in strain pIQ1 is a typical cpe-negative cpb2-negative type D plasmid [15].

Table 5. Comparisons between the reference plasmid sequences and plasmids constructed in silico using both the reference-based assembly (RBA) and assembly-graph (AG) approaches. REF: Reference sequences.

\begin{tabular}{ccccccccc}
\hline Strain & Plasmid & Method & Type & Size (bp) & Contig & GC (\%) & CDS & $\begin{array}{c}\text { Toxin Gene } \\
\text { Encoded }\end{array}$ \\
\hline \multirow{2}{*}{ IQ1 } & pIQ1a & RBA & D & 53,937 & 13 & 27.11 & 61 & etx \\
NCTC8533 & pCP8533etx & REF & D & 48,812 & 2 & 27.62 & 58 & etx \\
IQ2 & pIQ2a & RBA & E & 64,753 & 1 & 25.89 & 80 & etx, cpb2 \\
& pIQ2b & AG & E & 67,516 & 1 & 25.96 & 66 & iap, ibp, cpe \\
PB-1 & pCPPB-1 & REF & E & 67,479 & 1 & 25.97 & 66 & $i a p, i b p, c p e$ \\
IQ3 & pIQ3a & RBA & A & 54,460 & 1 & 25.05 & 53 & iap, ibp, cpe \\
OS-1 & pCP_OS1 & REF & A & 54,641 & 1 & 25.03 & 53 & bec A becB \\
\end{tabular}

Near-identical toxin-encoding plasmids were identified in both strains IQ2 and IQ3 with respect to known C. perfringens plasmids (Table 5; Figure 1B,C). The predicted conjugative plasmid pIQ2 was highly similar to the reference plasmid $\mathrm{PCPPB}-1$ (originally isolated from retail meat products in Japan); $\sim 67 \mathrm{~kb}$ in length, assembled into one full contig (GC $\sim 26 \%$ ), and encoding both iota toxin gene components and the enterotoxin gene cpe [24]. The predicted plasmid PIQ3 appeared near-identical to the reference plasmid pCP-OS1(originally from a clinical C. perfringens strain isolated from a diarrhoeal patient in Osaka, Japan) at a plasmid length $\sim 54 \mathrm{~kb}$, GC content $\sim 25 \%$ and assembled into one full contig, carrying intact bec $A B$ genes [19].

\subsection{Phylogenetic Analysis}

To understand the phylogenetic positions of these novel isolates, we constructed a core-genome based phylogenetic tree of 132 C. perfringens genomes, including all available 128 C. perfringens genome assemblies from the NCBI RefSeq database (July 2019). The type E isolate IQ2 was shown to be closely related, although not identical, to the French type E strains cp515.17 and cp508.17 (pairwise genetic distance: 649 SNPs, Figure 2). The BEC-positive isolate IQ3 was also found within the same clade as the type E isolates, which may indicate a geographical relatedness. Furthermore, the type D isolate IQ1, which carries etx, was located within a C. perfringens clade that comprises the type strain ATCC13124, and other type D strains including JGS1721 and NCTC8503.

Notably, the alveolysin toxin gene alv was demonstrated to be clade-specific (Figure 2), and thus likely to be chromosomally encoded. This clade consisted mainly of foal- and dog-associated type $\mathrm{F}$ isolates that encode the pore-forming toxin gene netF, alongside three type $\mathrm{E}$ isolates: cp515.17, cp508.17, IQ2 and BEC-positive isolate IQ3 [26]. 

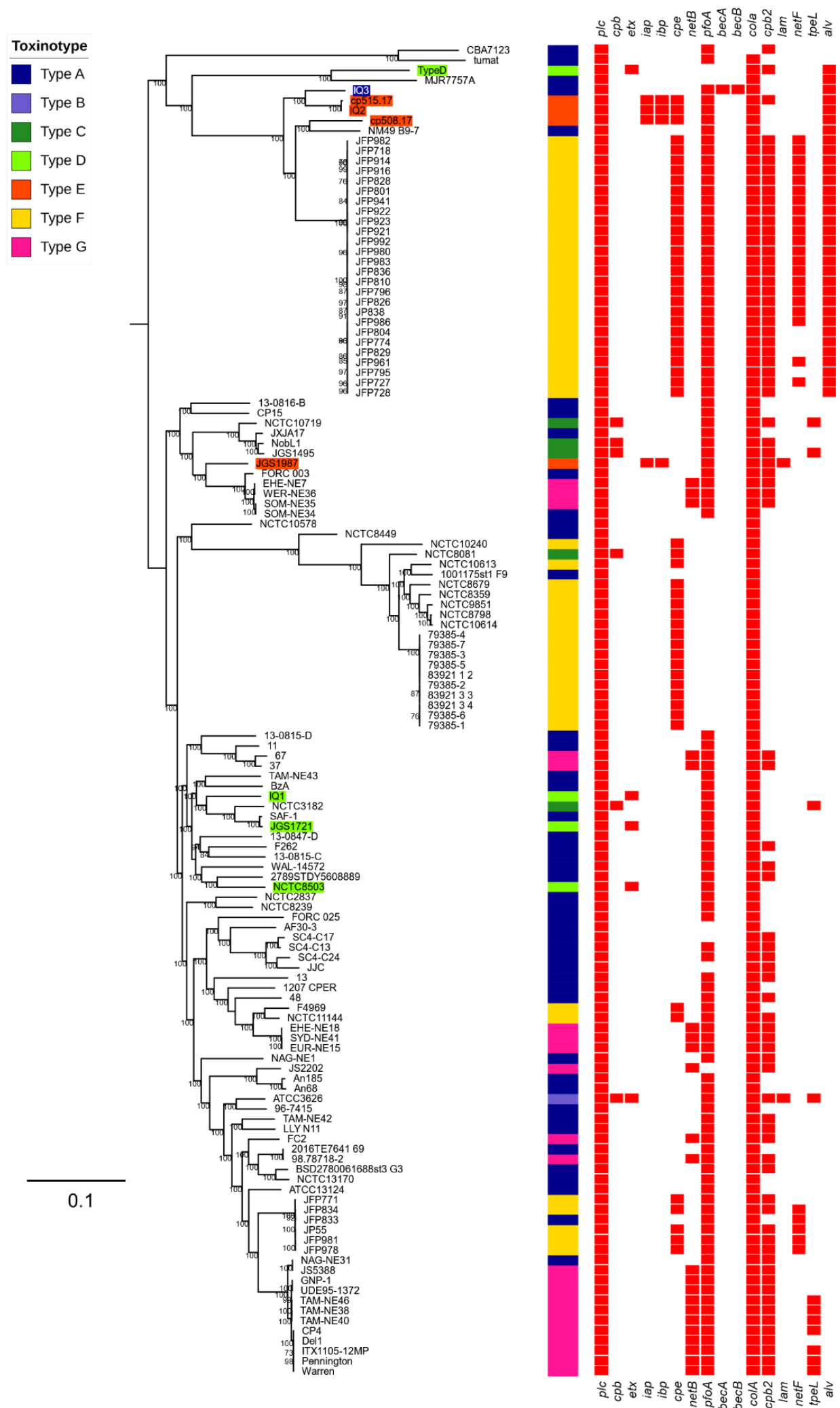

Figure 2. Maximum likelihood tree of 132 C. perfringens genomes constructed based on 115,533 SNPs aligned with the presence and absence of toxin genes and toxinotyping profiles (potential recent recombinations were removed). Bootstrap values $>70 \%$ are shown on the nodes. The presence of a gene is indicated by a red cell. Toxinotype D and E, and BEC/CPILE-positive C. perfringens strains are colour-labelled. 


\section{Discussion}

Conventionally, C. perfringens toxinotype $\mathrm{D}$ and $\mathrm{E}$ isolates are associated with diseases in domestic animals, including sheep and cattle, and rarely in humans $[7,18]$. To our knowledge, this is the first study that has reported, sequenced and analysed C. perfringens toxinotype D and $\mathrm{E}$ strains isolated from healthy children, alongside a BEC-positive isolate. We applied bioinformatics to analyse these isolates, including understanding key toxin gene variants, toxin-encoding plasmids and phylogenetic relatedness.

Previously, four C. perfringens type E strains (Japan, 2011) were reported to encode iota toxin genes on a pCPPB-1 plasmid ( $67 \mathrm{~kb})$, which also encoded a functional cpe gene (strains isolated from retail meat products) [24]. We also identified a near-identical pCPPB-1 plasmid, in our C. perfringens type $\mathrm{E}$ strain IQ2, which was surprising given that the isolate was obtained from an otherwise healthy child from the UK. Both iota-toxin gene components were identical to those in the French type E isolate cp515.17 (isolated from a 60-year-old woman, subsequent to food poisoning) and similar to cp508.17 (which originated from a calf), which are both known to carry a pCPPB-1 plasmid [21]. This suggests that this specific iota-toxin encoded plasmid is widely disseminated, or that it potentially shares a common source [21]. From a disease outbreak perspective, the conjugative nature of this plasmid may allow for a transfer to other $C$. perfringens strains in the same environmental niche; however, further surveys of diseased and healthy individuals (animals and humans) coupled with experimental studies are required to probe these insights further [27].

More recently, BEC-positive C. perfringens isolates were reported in Japanese gastroenteritis outbreaks, with this binary toxin experimentally proven to be toxigenic, thus highlighting the clinical importance of this toxin in the context of public health surveillance [19]. Notably, we observed a near-identical plasmid to pCPOS-1 (from the Japanese strain OS-1), carrying identical bec $A B$, in strain IQ3. This indicates that this plasmid may be more universally distributed than was first thought, which includes carriage in otherwise healthy children. Notably, a recent BEC-positive isolate obtained from a human adult was reported in Japan; however, the BEC genes $b e c A$ and $b e c B$ have a reduced homology, when compared to the isolate IQ3 (100\%) at $98.5 \%$ and $97.9 \%$ when compared to the reported strains TS1, OS1 and W5052 [20]. The possibility of a horizontal transfer of this plasmid should be investigated as no obvious conjugative system was identified.

As there is currently a limited set of $C$. perfringens whole genome sequences, this made the phylogenetic and genome-wide profiling of type $\mathrm{D}$ strains that carry the deadly epsilon toxin difficult. We were not able to completely predict the epsilon-toxin encoding plasmid, in part due to the unsuccessful assembly of repeated insertion sequences in the plasmid genome. Further experimental work to complement in silico studies is required, particularly phenotypic validations probing the expression profiles of toxin genes.

In addition to well described toxins, we also detected alveolysin, a thiol-activated (cholesterol-binding) pore-forming toxin first identified in Paenibacillus alvei (a beehive-colonising bacterium). This more 'uncommon' toxin is known to damage cell membranes in a mechanism that resembles the homologous thiol-activated toxin perfringolysin $\mathrm{O}$ (theta toxin), which is typically produced by $C$. perfringens $[22,28]$. Importantly, membrane-damaging alveolysin also shares sequence homologies with other pore-forming toxins secreted by other Gram-positive bacteria, including listeriolysin O, pneumolysin and streptolysin O [22,29]. Despite the potential importance of alveolysin in C. perfringens, this toxin has received very limited research attention over the past 20 years. Its unique clade-specificity and association with canine/foal enteritis, in both type D and type E isolates, highlights the need for further research, particularly with respect to intestinal diseases in domestic animals.

The strains described in this study were isolated as a part of a longitudinal study of the developing gastrointestinal microbiota in preterm infants [30]. Thus, it may be that the acquisition of these strains by healthy two-year-old children (residing at home) may have originally occurred during their stay in neonatal intensive care units. However, based on our larger-scale preterm C. perfringens screening study (unpublished data), we have not detected any other type D or E C. perfringens in samples collected 
from these infants earlier in life, or indeed in any of the other $>350$ preterm infants, suggesting that these strains (and plasmids) are not hospital-acquired and are not widely-circulating.

Importantly, the fact that these toxigenic $C$. perfringens isolates were obtained from healthy individuals indicated that other factors may predispose individuals to symptomatic illness. As these are gut-associated C. perfringens strains, the microbiota would be expected to play a significant colonisation resistance role by limiting access to nutrients and/or via direct antagonism through the production of antimicrobials. These toxigenic gut pathogens could exist within the wider microbial ecosystem without overgrowing or expressing toxin genes. However, disturbances of the resident microbiota by, e.g., antibiotics, could circumvent these protective responses, which may lead to overt disease symptoms. Further studies probing the role of the early life microbiota in this context may reveal underlying mechanisms maintaining the carriage of these potential deadly toxin types in the wider population [31,32].

\section{Conclusions}

Our analysis indicates the clade-specificity of the putative toxin gene alv (alveolysin), which was also encoded in the isolates IQ2 and IQ3. Importantly, the near-identical pCPPB-1 plasmid (previously reported in type E strains from Japan and France) was detected in the isolate IQ2, while the pCPOS-1 plasmid (previously associated with gastroenteritis outbreaks in Japan) was encoded in the isolate IQ3, highlighting a potential common source of these $C$. perfringens-derived plasmids, or otherwise a probable global distribution. Furthermore, a close phylogenetic relatedness was inferred between the French type E isolates cp515.17 and IQ2 in this study, suggesting geographical links. Whilst, WGS and a bioinformatic analysis has allowed us to probe the phylogenetic relatedness and global dissemination of three novel C. perfringens strains, the further large-scale sequencing of isolates from various toxinotypes is required to more accurately determine the spread of strains harboring disease-relevant toxins. Further experimental work is also required to probe toxin-disease mechanisms and complement any large-scale screening studies for the development of effective clinical and veterinary health surveillance strategies.

\section{Materials and Methods}

\subsection{Ethics Declaration}

The initial NeoM study ("Defining the Intestinal Microbiota in Premature Infants") was approved by West London Research Ethics Committee Two, United Kingdom (reference number 10/H0711/39). Parents gave written informed consent for their infant to participate in the study.

The NeoM follow-up study ("The Microbiota of the Premature Neonatal Gastrointestinal Tract: its development from birth to early childhood") was approved by the National Research Ethics Service Committee London-Chelsea, United Kingdom (reference number 13/LO/0693). Parents gave written informed consent for their child to participate in the study. Date of approval: 2 July 2013

\subsection{Sample Collection}

NeoM follow-up study: Faecal samples were collected at home by the parents/carers of the participants and placed into a sterile faecal collection pot using the attached sterile scoop. This was posted to the laboratory ideally within $24 \mathrm{~h}$ and immediately aliquoted and stored in DNAase-, RNAase-free Eppendorf tubes at $-80{ }^{\circ} \mathrm{C}$ upon receipt.

\subsection{Bacterial Isolation, Identification and Storage}

Twenty-five milligrams of freshly thawed faeces were added to $0.5 \mathrm{~mL}$ of Robertson's Cooked Meat Media broth. Five hundred microliters of $100 \%$ ethanol was added to the broth, vortexed for ten seconds and incubated for 30 minutes at room temperature. The broth was streaked on to fastidious anaerobic agar with $0.1 \%$ sodium taurocholate hydrate and $5 \%$ defibrinated sheep blood and incubated at $37^{\circ} \mathrm{C}$ for $48 \mathrm{~h}$ in anaerobic conditions. Purity plates were grown for each 
morphologically distinct colony and for single colonies used for the species level identification by matrix-assisted laser desorption/ionization-time of flight (MALDI-TOF) using a Bruker Microflex LT (Bruker Daltonics). Stocks were stored in 70\% brain heart infusion (BHI) broth (Oxoid) and 30\% glycerol (Sigma) (Gillingham, UK) at $-80{ }^{\circ} \mathrm{C}$.

\subsection{Genomic DNA Extraction and Whole Genome Sequencing}

The bacterial isolates were cultured in BHI media anaerobically at $37{ }^{\circ} \mathrm{C}$ for $5-7 \mathrm{~h}$ prior to the DNA extraction. The genomic DNA extraction of the pure cultures was performed using a Phenol-Chloroform methodology, as described previously [33]. Sequencing was run on an Illumina HiSeq 2500 system with a paired-end insertion of length $125 \mathrm{bp}$. The sequencing coverage achieved an average of 207-fold for three novel C. perfringens genomes.

\subsection{Genome Assembly and Annotation}

All raw sequencing reads (FASTQ) were quality-trimmed and adapter-removed using TrimGalore v0.4.2 options -q 20 -phred33 followed by de novo assembly using SPAdes v3.11.1 [34]. The genome assembly was carried out using options $-\mathrm{k} 75,85,95,105$ (paired-end reads insert size $2 \times 125 \mathrm{bp}$ ) and -careful, followed by removing contigs with $<300 \mathrm{bp}$ using the in-house script. The assembly statistics were calculated using the custom Perl script, and all assembly sequences were contamination-checked using webtool ContEst16S to contain only a single 16S rRNA gene [35]. All assembled genomes were ensured to have ANI > 95\% (using fastANI v1.1) and dDDH > 70\% (using GGDC v2.1) with respect to the $C$. perfringens type strain ATCC13124 genome for the species validation [36,37]. All genomes were annotated using Prokka v1.13 with the specific Clostridium genus database (35 Clostridium species from NCBI RefSeq annotations; Table S5) with the parameters -usegenus -mincontiglen 300 [38].

The PacBio sequencing reads of the NCTC8503 genome were retrieved from the ENA database, and the genome was reconstructed using Canu v1.5 and optimised by Circlator v1.5.5 for comparison with novel strains in this study $[39,40]$.

\subsection{Genomic Analysis}

ARIBA was used to assemble the toxin-encoding plasmids from sequencing reads via a custom reference plasmid sequence database (at default parameters; Table S6), and most complete sequence clusters (by sequence length) were identified for subsequent analyses [41,42]. In addition, Bandage v0.8.1 was used to investigate and extract circular sequences manually identified in the de novo assembly graphs generated by Spades v3.11.1 [43]. Plasmid sequences were annotated using Prokka 1.13. Linear plasmid maps were visualised using Easyfig v2.2.2 [44]. Annotated GenBank files were colour-labelled using the Artemis genome browser [45].

The toxin and antimicrobial gene identifications were performed using ABRricate v0.8.11 based on a custom toxin database (Table S7) and the Comprehensive Antibiotic Resistance Database (CARD) v3.0.1, respectively, with options - minid $=90$ and - mincov $=80[42,46,47]$. MUSCLE v3.8.31 was used to align sequences prior to the base-by-base average sequence identity (\%) calculation using Panito v0.0.1 [48]. The base differences were calculated using snp-dists [49].

\subsection{Phylogenetic Analysis}

We used ParSNP v1.2 to reconstruct the Maximum Likelihood phylogenetic tree from 132 genomes (including 128 genomes batch-retrieved from the NCBI RefSeq database; July 2019; Table S8) with the type strain ATCC13124 as the reference genome, and potential recent recombinations were removed using -x option [50,51]. The phylogenetic trees were annotated using iTOL [52]. To identify the genetic distance, snp-sites v2.3.3 and snp-dists v0.2 were using to estimate the SNP differences in the SNP alignment $[49,53]$. 


\subsection{Data Deposition}

The raw sequencing reads and genome assemblies in this study were deposited in the European Nucleotide Archive (ENA) under project PRJEB33762. An interactive annotated phylogenetic tree (Figure 2) can be viewed at https://itol.embl.de/tree/149155196252188971562847462.

Supplementary Materials: The following are available online at http://www.mdpi.com/2072-6651/11/9/543/s1, Table S1: Clinical data on individual preterm infants from which C. perfringens strains were isolated, Table S2: Variable sites comparison in 864 amino acid multi-sequence alignments in between C. perfringens type E strains, Table S3: Blastn query coverage and identity data on BEC-positive C. perfringens IQ3 genome assembly using reference $b e c A B$ genes via ABRicate, Table S4: Identity of alveolysin gene alv in C. perfringens isolate IQ3, Table S5: RefSeq genomes used to generate Clostridium genus-specific database for genome annotation, Table S6: Accession numbers of plasmid sequences used in this study, Table S7: Accession numbers of toxin genes used in this study, Table S8: C. perfringens genomes retrieved from NCBI RefSeq database for phylogenetic analysis.

Author Contributions: Conceptualisation, R.K., L.J.H. and J.S.K.; methodology, R.K.; software, R.K.; validation, L.J.H.; formal analysis, R.K.; investigation, A.S., K.S., E.C. and R.K.; resources, J.S.K. and D.P.; data curation, K.S., A.S. and E.C..; writing-original draft preparation, R.K and L.J.H.; writing-review and editing, R.K., K.S., A.S., J.S.K. and L.J.H.; visualisation, R.K.; supervision, L.J.H.; project administration, R.K., D.P., K.S., J.S.K. and L.J.H.; funding acquisition, L.J.H. and J.S.K.

Funding: This work was supported by a Wellcome Trust Investigator Award (100974/C/13/Z), and the Biotechnology and Biological Sciences Research Council (BBSRC); Institute Strategic Programme Gut Microbes and Health BB/R012490/1, and its constituent project(s) BBS/E/F/000PR10353 and BBS/E/F/000PR10356 to Lindsay J Hall; and by funding from the Winnicott Foundation and the National Institute for Health Research (NIHR) Biomedical Research Centre based at Imperial Healthcare National Health Service (NHS) Trust and Imperial College London (to J. Simon Kroll). Kathleen Sim was funded during this work by an NIHR Doctoral Research Fellowship [NIHR-DRF-2011-04-128]. This article presents independent research funded by the NIHR. The views expressed are those of the authors and not necessarily those of the NHS, NIHR, the Department of Health or other funders.

Acknowledgments: This research was supported in part by the NBI Computing infrastructure for Science (CiS) group through the provision of a High-Performance Computing (HPC) Cluster. We thank Shabhonam Caim for his computational assistance. We also thank the sequencing team at Wellcome Trust Sanger Institute for the sequencing work.

Conflicts of Interest: The authors declare no conflict of interest. The funders had no role in the design of the study; in the collection, analyses, or interpretation of data; in the writing of the manuscript, or in the decision to publish the results.

\section{Abbreviations}

ANI, Average Nucleotide Identity; BEC, Binary Enterotoxin of Clostridium perfringens; CPILE, Clostridium perfringens Iota-Like Enterotoxin; CPE, Clostridium perfringens enterotoxin

\section{References}

1. Kiu, R.; Hall, L.J. An update on the human and animal enteric pathogen Clostridium perfringens. Emerg. Microbes Infect. 2018, 7, 141. [CrossRef] [PubMed]

2. Lebrun, M.; Mainil, J.G.; Linden, A. Cattle enterotoxaemia and Clostridium perfringens: Description, diagnosis and prophylaxis. Vet. Rec. 2010, 167, 13-22. [CrossRef] [PubMed]

3. Prescott, J.F.; Parreira, V.R.; Mehdizadeh Gohari, I.; Lepp, D.; Gong, J. The pathogenesis of necrotic enteritis in chickens: What we know and what we need to know: A review. Avian Pathol. 2016, 45, 288-294. [CrossRef] [PubMed]

4. Awad, M.M.; Ellemor, D.M.; Boyd, R.L.; Emmins, J.J.; Rood, J.I. Synergistic effects of alpha-toxin and perfringolysin $\mathrm{O}$ in Clostridium perfringens-mediated gas gangrene. Infect. Immun. 2001, 69, 7904-7910. [CrossRef] [PubMed]

5. Rood, J.I.; Adams, V.; Lacey, J.; Lyras, D.; McClane, B.A.; Melville, S.B.; Moore, R.J.; Popoff, M.R.; Sarker, M.R.; Songer, J.G. Expansion of the Clostridium perfringens toxin-based typing scheme. Anaerobe 2018. [CrossRef] [PubMed]

6. Petit, L.; Gibert, M.; Popoff, M. Clostridium perfringens: Toxinotype and genotype. Trends Microbiol. 1999, 7, 104-110. [CrossRef] 
7. Layana, J.E.; Fernandez Miyakawa, M.E.; Uzal, F.A. Evaluation of different fluids for detection of Clostridium perfringens type D epsilon toxin in sheep with experimental enterotoxemia. Anaerobe 2006, 12, $204-206$. [CrossRef]

8. Filho, E.J.; Carvalho, A.U.; Assis, R.A.; Lobato, F.F.; Rachid, M.A.; Carvalho, A.A.; Ferreira, P.M.; Nascimento, R.A.; Fernandes, A.A.; Vidal, J.E.; et al. Clinicopathologic features of experimental Clostridium perfringens type D enterotoxemia in cattle. Vet. Pathol. 2009, 46, 1213-1220. [CrossRef]

9. Muth, O.H.; Morrill, D.R. Control of enterotoxemia (pulpy kidney disease) in lambs by the use of alum precipitated toxoid. Am. J. Vet. Res. 1946, 7, 355-357.

10. Fernandez-Miyakawa, M.E.; Sayeed, S.; Fisher, D.J.; Poon, R.; Adams, V.; Rood, J.I.; McClane, B.A.; Saputo, J.; Uzal, F.A. Development and application of an oral challenge mouse model for studying Clostridium perfringens type D infection. Infect. Immun. 2007, 75, 4282-4288. [CrossRef]

11. Baker, I.; Van Dreumel, A.; PALMER, N. Chapter I, The Alimentary System. Pathol. Domest. Anim. 1993, 2, 141-144.

12. Finnie, J.W.; Blumbergs, P.C.; Manavis, J. Neuronal damage produced in rat brains by Clostridium perfringens type D epsilon toxin. J. Comp. Pathol. 1999, 120, 415-420. [CrossRef] [PubMed]

13. Tamai, E.; Ishida, T.; Miyata, S.; Matsushita, O.; Suda, H.; Kobayashi, S.; Sonobe, H.; Okabe, A. Accumulation of Clostridium perfringens epsilon-toxin in the mouse kidney and its possible biological significance. Infect. Immun. 2003, 71, 5371-5375. [CrossRef] [PubMed]

14. Smedley, J.G., 3rd; Fisher, D.J.; Sayeed, S.; Chakrabarti, G.; McClane, B.A. The enteric toxins of Clostridium perfringens. Rev. Physiol. Biochem. Pharmacol. 2004, 152, 183-204. [CrossRef] [PubMed]

15. Sayeed, S.; Li, J.; McClane, B.A. Virulence plasmid diversity in Clostridium perfringens type D isolates. Infect. Immun. 2007, 75, 2391-2398. [CrossRef]

16. McClane, B.A.; Uzal, F.A.; Miyakawa, M.E.F.; Lyerly, D.; Wilkins, T. The enterotoxic Clostridia. In The Prokaryotes: A Handbook on the Biology of Bacteria; Dworkin, M., Falkow, S., Rosenberg, E., Schleifer, K.H., Stackebandt, E., Eds.; Springer: Singapore, 2006; Volume 4, pp. 698-752.

17. Uzal, F.A.; Vidal, J.E.; McClane, B.A.; Gurjar, A.A. Clostridium Perfringens Toxins Involved in Mammalian Veterinary Diseases. Open Toxinol. J. 2010, 2, 24-42. [CrossRef]

18. Li, J.; Miyamoto, K.; McClane, B.A. Comparison of virulence plasmids among Clostridium perfringens type E isolates. Infect. Immun. 2007, 75, 1811-1819. [CrossRef]

19. Yonogi, S.; Matsuda, S.; Kawai, T.; Yoda, T.; Harada, T.; Kumeda, Y.; Gotoh, K.; Hiyoshi, H.; Nakamura, S.; Kodama, T.; et al. BEC, a novel enterotoxin of Clostridium perfringens found in human clinical isolates from acute gastroenteritis outbreaks. Infect. Immun. 2014, 82, 2390-2399. [CrossRef]

20. Matsuda, A.; Aung, M.S.; Urushibara, N.; Kawaguchiya, M.; Sumi, A.; Nakamura, M.; Horino, Y.; Ito, M.; Habadera, S.; Kobayashi, N. Prevalence and Genetic Diversity of Toxin Genes in Clinical Isolates of Clostridium perfringens: Coexistence of Alpha-Toxin Variant and Binary Enterotoxin Genes (bec/cpile). Toxins 2019, 11, 326. [CrossRef]

21. Diancourt, L.; Sautereau, J.; Criscuolo, A.; Popoff, M.R. Two Clostridium perfringens Type E Isolates in France. Toxins 2019, 11, 138. [CrossRef]

22. Geoffroy, C.; Mengaud, J.; Alouf, J.E.; Cossart, P. Alveolysin, the thiol-activated toxin of Bacillus alvei, is homologous to listeriolysin $\mathrm{O}$, perfringolysin $\mathrm{O}$, pneumolysin, and streptolysin $\mathrm{O}$ and contains a single cysteine. J. Bacteriol. 1990, 172, 7301-7305. [CrossRef] [PubMed]

23. Li, J.; Adams, V.; Bannam, T.L.; Miyamoto, K.; Garcia, J.P.; Uzal, F.A.; Rood, J.I.; McClane, B.A. Toxin plasmids of Clostridium perfringens. Microbiol. Mol. Biol. Rev. 2013, 77, 208-233. [CrossRef] [PubMed]

24. Miyamoto, K.; Yumine, N.; Mimura, K.; Nagahama, M.; Li, J.; McClane, B.A.; Akimoto, S. Identification of novel Clostridium perfringens type $\mathrm{E}$ strains that carry an iota toxin plasmid with a functional enterotoxin gene. PLoS ONE 2011, 6, e20376. [CrossRef] [PubMed]

25. Miyamoto, K.; Li, J.; Sayeed, S.; Akimoto, S.; McClane, B.A. Sequencing and diversity analyses reveal extensive similarities between some epsilon-toxin-encoding plasmids and the pCPF5603 Clostridium perfringens enterotoxin plasmid. J. Bacteriol. 2008, 190, 7178-7188. [CrossRef] [PubMed]

26. Gohari, I.M.; Kropinski, A.M.; Weese, S.J.; Whitehead, A.E.; Parreira, V.R.; Boerlin, P.; Prescott, J.F. NetF-producing Clostridium perfringens: Clonality and plasmid pathogenicity loci analysis. Infect. Genet. Evol. 2017, 49, 32-38. [CrossRef] [PubMed] 
27. Brynestad, S.; Sarker, M.R.; McClane, B.A.; Granum, P.E.; Rood, J.I. Enterotoxin plasmid from Clostridium perfringens is conjugative. Infect. Immun. 2001, 69, 3483-3487. [CrossRef] [PubMed]

28. Thelestam, M.; Alouf, J.E.; Geoffroy, C.; Mollby, R. Membrane-damaging action of alveolysin from Bacillus alvei. Infect. Immun. 1981, 32, 1187-1192. [PubMed]

29. Rossjohn, J.; Gilbert, R.J.; Crane, D.; Morgan, P.J.; Mitchell, T.J.; Rowe, A.J.; Andrew, P.W.; Paton, J.C.; Tweten, R.K.; Parker, M.W. The molecular mechanism of pneumolysin, a virulence factor from Streptococcus pneumoniae. J. Mol. Biol. 1998, 284, 449-461. [CrossRef]

30. Sim, K.; Shaw, A.G.; Randell, P.; Cox, M.J.; McClure, Z.E.; Li, M.S.; Haddad, M.; Langford, P.R.; Cookson, W.O.; Moffatt, M.F.; et al. Dysbiosis anticipating necrotizing enterocolitis in very premature infants. Clin. Infect. Dis. 2015, 60, 389-397. [CrossRef]

31. Pickard, J.M.; Zeng, M.Y.; Caruso, R.; Nunez, G. Gut microbiota: Role in pathogen colonization, immune responses, and inflammatory disease. Immunol. Rev. 2017, 279, 70-89. [CrossRef]

32. Lawley, T.D.; Walker, A.W. Intestinal colonization resistance. Immunology 2013, 138, 1-11. [CrossRef] [PubMed]

33. Kiu, R.; Caim, S.; Alcon-Giner, C.; Belteki, G.; Clarke, P.; Pickard, D.; Dougan, G.; Hall, L.J. Preterm Infant-Associated Clostridium tertium, Clostridium cadaveris, and Clostridium paraputrificum Strains: Genomic and Evolutionary Insights. Genome Biol. Evol. 2017, 9, 2707-2714. [CrossRef] [PubMed]

34. Bankevich, A.; Nurk, S.; Antipov, D.; Gurevich, A.A.; Dvorkin, M.; Kulikov, A.S.; Lesin, V.M.; Nikolenko, S.I.; Pham, S.; Prjibelski, A.D.; et al. SPAdes: A new genome assembly algorithm and its applications to single-cell sequencing. J. Comput. Biol. 2012, 19, 455-477. [CrossRef] [PubMed]

35. Lee, I.; Chalita, M.; Ha, S.M.; Na, S.I.; Yoon, S.H.; Chun, J. ContEst16S: An algorithm that identifies contaminated prokaryotic genomes using 16S RNA gene sequences. Int. J. Syst. Evol. Microbiol. 2017, 67, 2053-2057. [CrossRef] [PubMed]

36. Jain, C.; Rodriguez, R.L.; Phillippy, A.M.; Konstantinidis, K.T.; Aluru, S. High throughput ANI analysis of $90 \mathrm{~K}$ prokaryotic genomes reveals clear species boundaries. Nat. Commun. 2018, 9, 5114. [CrossRef] [PubMed]

37. Meier-Kolthoff, J.P.; Auch, A.F.; Klenk, H.P.; Goker, M. Genome sequence-based species delimitation with confidence intervals and improved distance functions. BMC Bioinforma. 2013, 14, 60. [CrossRef] [PubMed]

38. Seemann, T. Prokka: Rapid prokaryotic genome annotation. Bioinformatics 2014, 30, 2068-2069. [CrossRef] [PubMed]

39. Koren, S.; Walenz, B.P.; Berlin, K.; Miller, J.R.; Bergman, N.H.; Phillippy, A.M. Canu: Scalable and accurate long-read assembly via adaptive k-mer weighting and repeat separation. Genome Res. 2017, 27, 722-736. [CrossRef]

40. Hunt, M.; Silva, N.D.; Otto, T.D.; Parkhill, J.; Keane, J.A.; Harris, S.R. Circlator: Automated circularization of genome assemblies using long sequencing reads. Genome Biol. 2015, 16, 294. [CrossRef] [PubMed]

41. Hunt, M.; Mather, A.E.; Sanchez-Buso, L.; Page, A.J.; Parkhill, J.; Keane, J.A.; Harris, S.R. ARIBA: Rapid antimicrobial resistance genotyping directly from sequencing reads. Microb Genom 2017, 3, e000131. [CrossRef]

42. Kiu, R.; Caim, S.; Alexander, S.; Pachori, P.; Hall, L.J. Probing Genomic Aspects of the Multi-Host Pathogen Clostridium perfringens Reveals Significant Pangenome Diversity, and a Diverse Array of Virulence Factors. Front. Microbiol. 2017, 8, 2485. [CrossRef] [PubMed]

43. Wick, R.R.; Schultz, M.B.; Zobel, J.; Holt, K.E. Bandage: Interactive visualization of de novo genome assemblies. Bioinformatics 2015, 31, 3350-3352. [CrossRef] [PubMed]

44. Sullivan, M.J.; Petty, N.K.; Beatson, S.A. Easyfig: A genome comparison visualizer. Bioinformatics 2011, 27, 1009-1010. [CrossRef] [PubMed]

45. Rutherford, K.; Parkhill, J.; Crook, J.; Horsnell, T.; Rice, P.; Rajandream, M.A.; Barrell, B. Artemis: Sequence visualization and annotation. Bioinformatics 2000, 16, 944-945. [CrossRef] [PubMed]

46. Seemann, T. ABRicate: Mass screening of contigs for antimicrobial and virulence genes. Available online: https://github.com/tseemann/abricate (accessed on 2 August 2019).

47. Jia, B.; Raphenya, A.R.; Alcock, B.; Waglechner, N.; Guo, P.; Tsang, K.K.; Lago, B.A.; Dave, B.M.; Pereira, S.; Sharma, A.N.; et al. CARD 2017: Expansion and model-centric curation of the comprehensive antibiotic resistance database. Nucleic Acids Res. 2017, 45, D566-D573. [CrossRef] [PubMed] 
48. Edgar, R.C. MUSCLE: Multiple sequence alignment with high accuracy and high throughput. Nucleic Acids Res. 2004, 32, 1792-1797. [CrossRef]

49. Seemann, T.; Klotzl, F.; Page, A.J. Snp-dists: Pairwise SNP distance matrix from a FASTA sequence alignment. Available online: https://github.com/tseemann/snp-dists (accessed on 2 August 2019).

50. Treangen, T.J.; Ondov, B.D.; Koren, S.; Phillippy, A.M. The Harvest suite for rapid core-genome alignment and visualization of thousands of intraspecific microbial genomes. Genome Biol. 2014, 15, 524. [CrossRef]

51. Bruen, T.C.; Philippe, H.; Bryant, D. A simple and robust statistical test for detecting the presence of recombination. Genetics 2006, 172, 2665-2681. [CrossRef]

52. Letunic, I.; Bork, P. Interactive tree of life (iTOL) v3: An online tool for the display and annotation of phylogenetic and other trees. Nucleic Acids Res. 2016, 44, W242-W245. [CrossRef]

53. Page, A.J.; Taylor, B.; Delaney, A.J.; Soares, J.; Seemann, T.; Keane, J.A.; Harris, S.R. SNP-sites: Rapid efficient extraction of SNPs from multi-FASTA alignments. Microb Genom. 2016, 2, e000056. [CrossRef]

(C) 2019 by the authors. Licensee MDPI, Basel, Switzerland. This article is an open access article distributed under the terms and conditions of the Creative Commons Attribution (CC BY) license (http://creativecommons.org/licenses/by/4.0/). 